\title{
Optical Features of Substituted Phenyl End-Capped Oligothiophenes
}

\author{
E.V. Canesi ${ }^{*}, 1$, G. Dassa ${ }^{1}$, C. Botta ${ }^{2}$, A. Bianco ${ }^{1}$, C. Bertarelli ${ }^{1}$ and G. Zerbi ${ }^{1}$

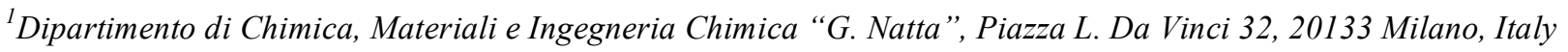 \\ ${ }^{2}$ ISMAC-CNR, Via E. Bassini 15, 20133 Milano, Italy
}

\begin{abstract}
A series of phenyl end-capped oligothiophenes has been synthesized and a study of the absorption and emission properties has been carried out; the photoluminescence quantum yield has been measured both in solution and in solid state. The characterization, supported by DFT calculations, highlights a strong relationship between the optical features and the differences in the chemical structure. The results show that some molecules are of interest in the field of organic light emitting diodes (OLEDs).
\end{abstract}

\section{INTRODUCTION}

Organic conjugated materials have been deeply investigated in the last years due to their applicability in electrical and optoelectronic devices.

Thiophene/phenyl co-oligomers have been already reported as semiconductors for organic transistors (TFTs) [1] or as emitting layers for OLEDs [2]. By changing the extent and the conformation of the oligothienyl moiety it is possible to efficiently tune the colour and $\pi$-conjugation length of the oligomers. Nevertheless, the control of molecular packing is of crucial importance in order to tailor their charge transfer and emission properties in the solid state. The solid state packing is desired in electric devices such as TFTs because it enhances the charge mobility, but it usually reduces the emissive response [3]. The addition of bulky terminal groups can prevent the formation of well-organized tridimensional structures, with a positive effect on the photoemissive properties in the solid state $[2,4,5]$.

The molecules investigated in this work show substituted phenyl groups added to the thiophene rings in two different positions: the study of the relationship between property and structure highlights that the position of the functional groups deeply modify the optical features of these materials. These findings have been associated to the conformational features of the ground and excited states.

The effect of the length of the thiophene moiety on the optical properties has also been investigated. The series of molecules is enriched considering some related quinoid structures.

Finally, the quantitative analysis of the emissive properties shows some materials that can be of interest for the realization of optoelectronic devices.

\section{SYNTHESIS}

The molecules involved in this study are divided in oligothiophenes with phenol in the $\alpha$ (Fig. 1a-d) or in the $\beta$

*Address correspondence to this author at the Dipartimento di Chimica, Materiali e Ingegneria Chimica "G. Natta", Piazza L. Da Vinci 32, 20133 Milano, Italy; E-mail: eleonora.canesi@chem.polimi.it
(Fig. 1e,f) position of the thiophene rings. The latters were synthesized in order to evaluate the effect of the steric hindrance on the optical properties: actually, we supposed for these structures a less planar geometry at the ground state. This feature would have a strong influence on the typical optical properties of thiophene-based materials [6].

The phenyl ring bears tert-butyl groups in -meta positions and a protected alcoholic group in -para position: the roles of the tert-butyl are to enhance the solubility, the stability and to further increase the steric hindrance of the functionality while the oxygen atom allows to yield the quinoid structures by oxidation of the correspondent aromatic ones. In the aromatic molecules, the alcoholic groups are protected with methyls, in order to improve their stability. In molecules $\mathbf{e}$ and $\mathbf{f}$, the positions 5, 5' of the bithiophenes are unsubstituted, making possible a subsequent polymerization reaction.

The aromatic molecules are synthesized by Suzuki coupling: for $\mathbf{e}$, the general pathway consists in the coupling of 1-methoxy-2,6-di-tert-butyl-4-bromo benzene [7] with 3thiophen boronic acid. The resulting intermediate is first brominated in 2-position with $\mathrm{N}$-bromosuccinimide in dimethylformamide and then reacts with buthyllithium and couples with $\mathrm{CuCl}_{2}$ to yield the targeted molecule. For a, b and c, 1-methoxy-2,6-di-tert-butyl-4-bromo benzene reacts via Suzuki with a suitable functionalized oligothiophene: 2,5-diboronic acid thiophene, 2,2'-bithiophen-5,5'-diboronic acid and 2,2':5',2':5',2,' '-tetrathiophen-5,5"'-diboronic acid respectively; the diboronic derivatives are synthesized through litiation of the respective di-bromo precursors in dry THF and subsequent quenching with trimethylborate.

To obtain the quinoid structures $(\mathbf{d}, \mathbf{f})$, the corresponding aromatic structures with deprotected hydroxyl are oxidized through $\mathrm{PbO}_{2}$. These aromatic structures are synthesized with the same procedure of $\mathbf{b}$ and $\mathbf{e}$, with $-\mathrm{OSiMe}_{3}$ instead of -OMe as protecting group; the protection is then removed through a treatment with tetra-n-butylammonium fluoride. For d, the synthesis here described is different from that previously reported in the literature $[8,9]$. 
<smiles>COc1c(C(C)(C)C)cc(-c2ccc(-c3cc(C(C)(C)C)c(OC)c(C(C)(C)C)c3)s2)cc1C(C)(C)C</smiles>

(a)<smiles>COc1c(C(C)(C)C)cc(-c2ccc(-c3ccc(-c4cc([15CH])c(C(C)(C)C)c([15CH]C(C)(C)C)c4)s3)s2)cc1C(C)(C)C</smiles>

(b)<smiles>CCC(C)c1cc(-c2ccsc2-c2sccc2-c2cc([13C](C)C)c(OC)c([14C](C)CC(C)(C)C)c2)cc(C(C)(C)C)c1OC</smiles>

(e)<smiles></smiles>

(c)<smiles>CC(C)C(=C(C1=CC(=c2ccc(=c3ccc(=C4C=C(C(C)(C)C)C(=O)C(C(C)(C)C)=C4)s3)s2)C=C(C(C)(C)C)C1=O)C(C)(C)C)C(C)(C)C</smiles>

(d)

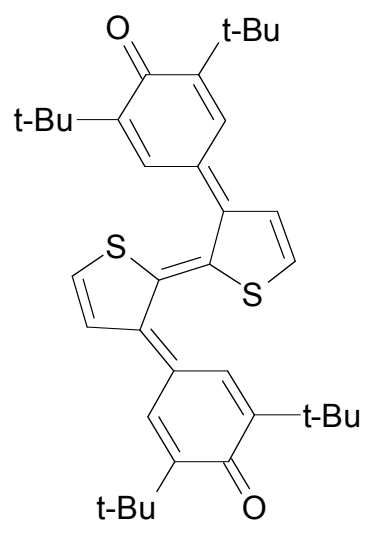

(f)

Fig. (1). Structure of the molecules: (a) 2,5-bis(3,5-di-tert-butyl-4-metoxyphenyl)thiophene (b) 5,5'-bis(3,5-di-tert-butyl-4metoxyphenyl) 2,2'bithiophene (c) 5,5','-bis(3,5-di-tert-butyl-4-metoxyphenyl)-2,2':5',2':5', ,','-tetrathiophene (d) 5,5'-bis(3,5-di-tert-butyl-4-oxo-2,5cicloesadien-1-yliden)-5,5'-dihydroxy-2,2'-bithiophene (e) 3,3'-bis(3,4-di-tert-butyl-4-metoxyphenyl)-2,2'-bithiophene (f) 3,3'-bis(3,5-ditert-butyl-4-oxo-2,5-cicloesadien-1-ylidien)-2,2'-dihydroxy-bithiophene.

Unless otherwise specified, all reagents and catalysts were commercial (Sigma Aldrich).

All the molecules have been characterized with ${ }^{1} \mathrm{H}$-NMR spectroscopy.

\section{RESULTS AND DISCUSSION}

Both absorption and photoluminescence (PL) analysis were carried out (Fig. 2a and $\mathbf{2 b}$, respectively). The spectra were collected on very dilute solutions $\left(<10^{-5} \mathrm{M}\right)$, as usual in fluorescence spectroscopy, in order to avoid aggregation phenomena and minimize internal filter effect. We report the photoluminescence data only for the aromatic molecules, because the emission of the quinoid ones is extremely low and strongly related to the exciting beam energy and to the solution concentration.

In Table 1, the wavelength maxima of absorption and emission spectra are reported, together with the measured absorption coefficients $(\varepsilon)$ and the PL quantum yields (QY). The values of the Stokes shift (obtained as the energy difference between the maximum of the absorption and the $0-0$ emission peak) are also reported.
The peculiar batochromic shift of the electronic transition going to longer oligothiophenes is mantained in the studied molecules, as shown by $\mathbf{a}, \mathbf{b}$ and $\mathbf{c}$ absorption spectra: thus, the presence of bulky groups in $\alpha$ does not prevent the delocalization among the thiophene chain. Furthermore, the delocalization involves also the phenol rings: actually, unsubstituted oligothiophenes absorptions are located at higher energies, respectively 242, 305 and $393 \mathrm{~nm}$ for molecules 1, 2, 3 of Fig. (3).

The photoluminescence spectra of molecules $\mathbf{a}, \mathbf{b}$ and $\mathbf{c}$ show well-defined vibronic peaks with energy separation of about $0.16 \mathrm{eV}$. The Stokes shift values are in agreement with those reported for similar molecules [2] and show a decrease for longer thiophene chains.

Very different features are shown by the molecule with phenyls in $\beta$ (e). First of all, the high energy of the $\pi-\pi^{*} a b-$ sorption reveals a low conjugation length in the ground state. The absorption spectrum of $\mathbf{e}$ is comparable with that of 
(a)

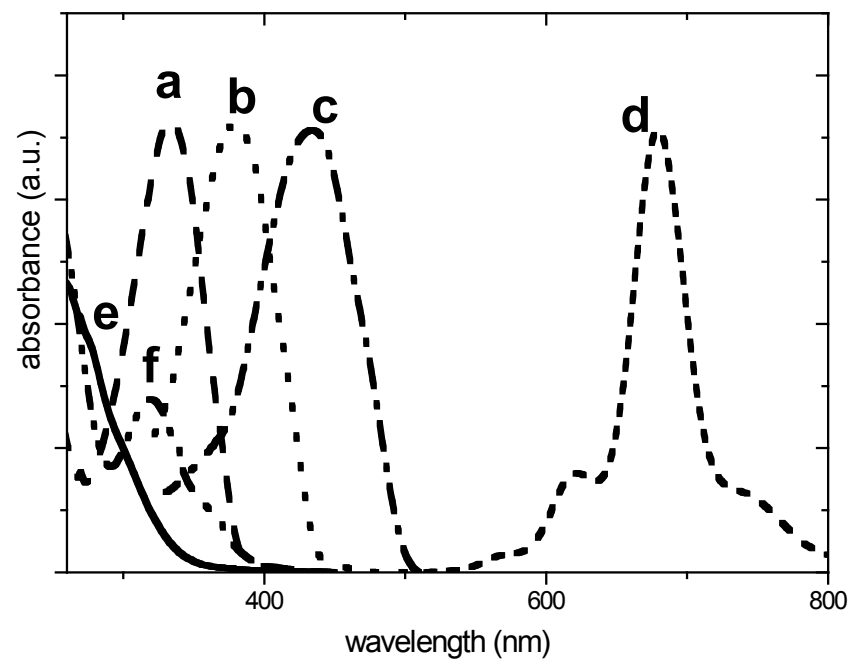

(b)

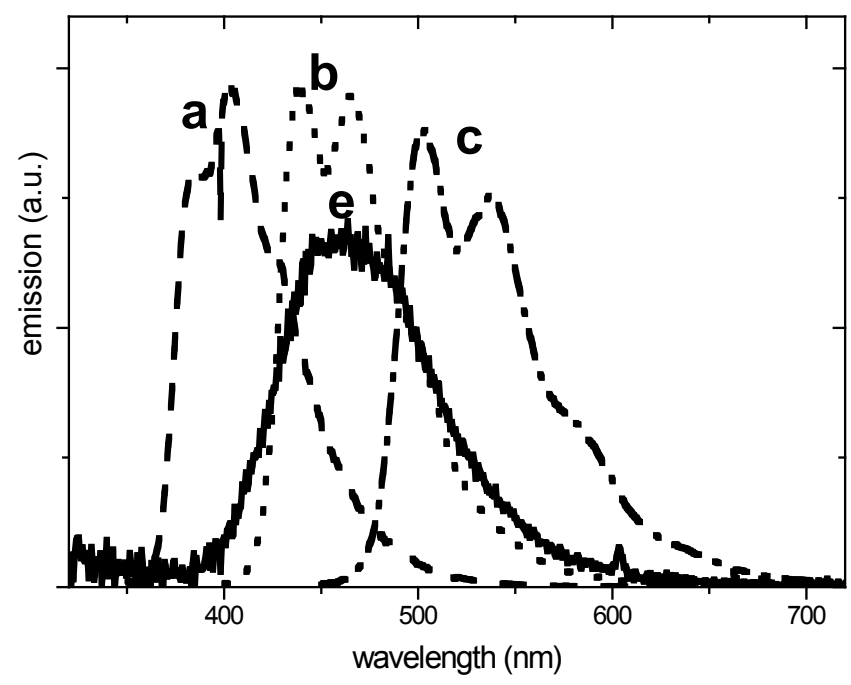

Fig. (2). (a) Absorption and (b) emission spectra in solution of: a (dash), b (dot), c (dash dot), d (short dash), e (solid), f (dash dot dot).

Table 1. Experimental Wavelength Positions of the Absorption and Photoluminescence Peaks in Solution, Absorption Coefficients, PL QY (Absolute Values) and Stokes Shift Values. Solvent: [a] THF, [b] $n$ Heptane, [c] Chloroform

\begin{tabular}{|c|c|c|c|c|c|}
\hline & $\begin{array}{c}\text { Abs } \\
(\mathbf{n m})\end{array}$ & $\boldsymbol{\varepsilon}\left(\mathbf{M}^{-1} \mathbf{c m}^{-\mathbf{1}}\right)$ & $\begin{array}{c}\mathbf{P L} \\
(\mathbf{n m})\end{array}$ & $\begin{array}{c}\mathbf{Q Y} \\
\mathbf{( \% )}\end{array}$ & $\begin{array}{c}\text { Stokes } \\
\text { Shift (eV) }\end{array}$ \\
\hline \hline $\mathrm{a}[\mathrm{a}]$ & 334 & 31000 & 385404427 & 15 & 0.49 \\
\hline $\mathrm{b}[\mathrm{a}]$ & 378 & 40000 & 438464495 & 16 & 0.45 \\
\hline $\mathrm{c}[\mathrm{a}]$ & 433 & 52000 & 502540583 & 56 & 0.39 \\
\hline $\mathrm{e}[\mathrm{b}]$ & 270 & 26000 & 460 & 4 & 1.90 \\
\hline $\mathrm{d}[\mathrm{c}]$ & 680 & 120000 & & & \\
\hline $\mathrm{f}[\mathrm{c}]$ & 320258 & 1200032000 & & & \\
\hline
\end{tabular}

molecule 4, that corresponds to half of e molecule: thus, in $\mathbf{e}$ the delocalization of $\pi$-electrons between the two thiophenes is almost completely interrupted. This evidence confirms the hypothesis that the steric groups in $\beta$ position generate a twisted molecular skeleton. Molecule e shows a much higher Stokes shift, indicating a relevant difference between the geometry of the ground and the excited states. Moreover, the PL spectra of $\mathbf{e}$ and $\mathbf{b}$ show similar position suggesting that in the excited state their geometry might have not a significant difference.

Both for molecules with phenyls in $\alpha$ or in $\beta$, the transition from aromatic to quinoid structures $(\mathbf{d}, \mathbf{f})$ is related to a shift of the absorption spectrum to higher wavelengths: the almost-double inter-ring bonds force the skeleton in a more planar conformation and a more extended delocalization is then achieved. This effect is of different entity for the two analyzed quinoid structures: the high energy absorption of $\mathbf{f}$ gives raise to the hypothesis that the planarization effect is balanced by the steric hindrance of the functional groups in $\beta$.

Density functional theory (DFT) calculations have been carried out on all the four diphenyl-bithiophenes, to determine how the substitution in $\alpha$ or in $\beta$ affects their geometrical conformation. The thiophene-thiophene torsional angle of the optimized geometries turned out to be of about $65^{\circ}$, $35^{\circ}, 20^{\circ}, 0^{\circ}$ for $\mathbf{e}, \mathbf{f}, \mathbf{b}, \mathbf{d}$ respectively. Thus, the presence of bulky groups in $\beta$ is responsible for a twist of the thienyl skeleton, and this effect is well evident also for the quinoid species (molecule $\mathbf{f}$ ); nevertheless, in both cases $(\alpha$ or $\beta$ ) going from the aromatic to the quinoid structures a planarization occurs. The completely planar conformation of molecule $\mathbf{d}$ is consistent with its very low absorption energy.

A quantitative analysis of the emissive properties has been also carried out, as a first indication of the applicability of these molecules in optoelectronic devices. The experiments were firstly performed in dilute solutions $\left(<10^{-5} \mathrm{M}\right)$ to get information of the intrinsic molecular property, avoiding aggregation and re-absorption phenomena; following the procedure in [10], the results reported in Table $\mathbf{1}$ were obtained.

The study of the quantum yields reveals again a strong difference between the molecules with phenyls in $\alpha$ or in $\beta$ : actually, the PL QY of $\mathbf{e}$ is lower with respect to that of the linear corresponding b. This difference can be explained through the Stokes shift values: an high Stokes shift implies that upon photoexcitation a large geometrical relaxation can introduce non radiative decay channels. As a result, molecules possessing non-planar ground state and high torsional freedom, might have lower radiative emission [11].

Molecules with phenyls in $\alpha$ mantain all the typical properties of oligothiophenes, such as the red-shift of the emission and the increase of the quantum yield with the chain length. In $\mathbf{a}, \mathbf{b}$ and $\mathbf{c}$ the presence of bulky phenyl groups enhances the quantum yields with respect to the unsubstituted oligothiophenes (QY of molecules $\mathbf{2}$ and $\mathbf{3}$ is about $2 \%$ and $18 \%$, respectively [12]). This finding can be related both to the increased conjugation length due to the extension 


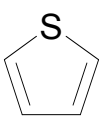

(1)

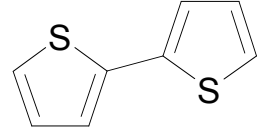

(2)

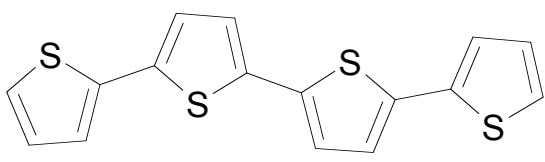

(3)

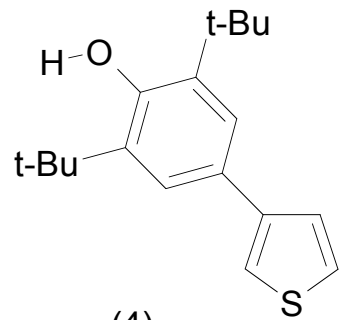

(4)

Fig. (3). Reference molecules.

(a)

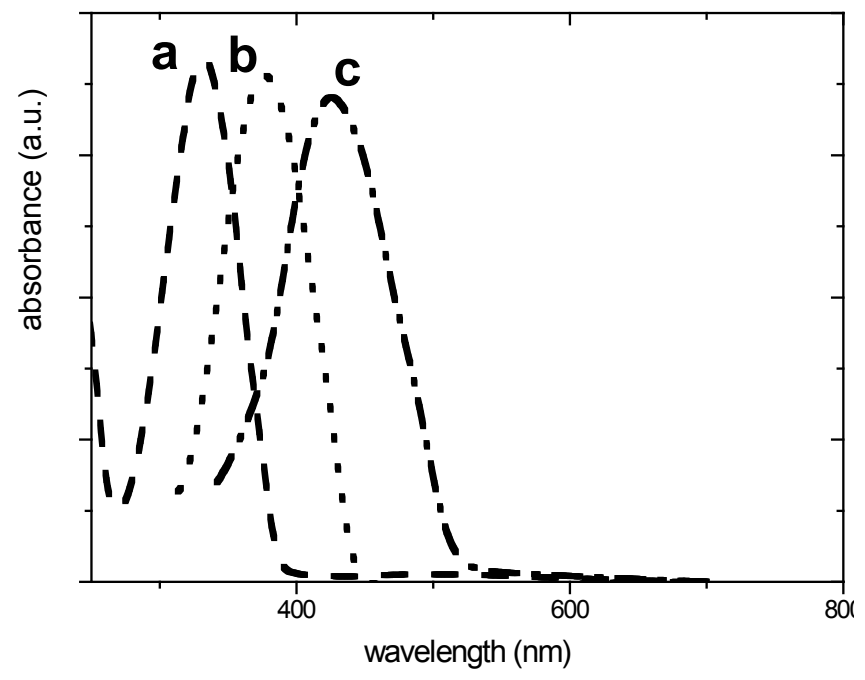

(b)

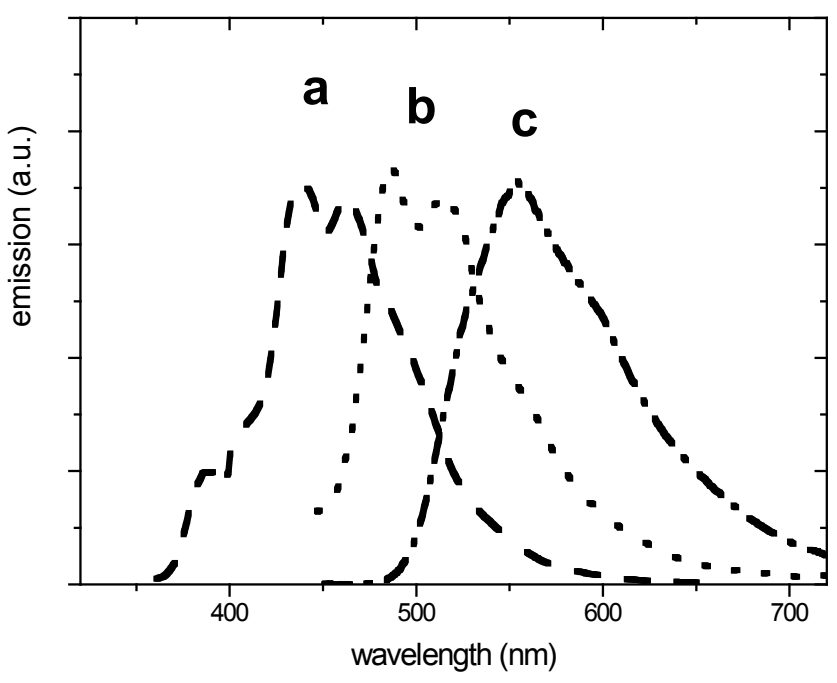

Fig. (4). (a) Absorption and (b) emission spectra of spin coating films of: a (dash), b (dot), c (dash dot).

of the $\pi$ electron delocalization on the phenyl rings, and to the intrinsically higher QY of the phenyl ring with respect to the thiophene ring [2].

We expected that the functional groups have also the positive effect of hinder an ordered packing of the molecules in the solid state. To evaluate this effect, solid state measurements of quantum yield have been carried out. The results are of importance for the applicability of these molecules in optical devices such as OLEDs, where a solid state material is required.

In Fig. (4), the absorption and emission spectra of the molecules $\mathbf{a}, \mathbf{b}$ and $\mathbf{c}$ at the solid state are reported. While the absorption spectra are very similar to those in solution, for photoluminescence a batochromic shift is observed for all the three molecules (the peaks move to 440, 487, $554 \mathrm{~nm}$, respectively). Moreover, for $\mathbf{c}$ the vibronic structure almost disappears.

In Table 2 we report the solid state PL quantum yields measured following the procedure described in the literature [13]. We analyzed samples obtained with different deposition techniques, and thus different degree of intermolecular organization at the solid state. Indeed, a slow evaporation of the solvent allows to obtain big crystals, while solution casting or spin coating leads to a faster solvent evaporation.

Table 2. Solid State PL Quantum Yields, Compared with Solution Data (\%)

\begin{tabular}{|c|c|c|c|}
\hline & a & b & c \\
\hline \hline solution & 15 & 16 & 56 \\
\hline spin coating & 12 & 19 & 6 \\
\hline casting & 17 & 23 & 11 \\
\hline crystals & 5 & 17 & 9 \\
\hline
\end{tabular}

Molecule c shows a strong quenching of the emission at the solid state. This feature has been widely reported for unsubstituted oligothiophenes as due to strong intermolecular interactions of the molecules assuming H-type aggregation. Due to the forbidden nature of the electronic transition from the lower excited state of these aggregates their PL QY is extremely low $[2,14]$. For molecule $\mathbf{c}$ the steric hindrance of the phenyl groups is secondary with respect to the tendency of molecular packing of the quaterthienyl moiety.

With shorter thiophene chains (a and b), the intermolecular interaction effect is less evident: the PL quantum yield of the films is equal or even higher than that in solution. For these molecules the steric hindrance of the phenyl groups prevent H-type aggregation and therefore PL quenching in the solid state. Both the higher QYs and the red-shifted PL indicate that the $\mathbf{a}$ and $\mathbf{b}$ emitting molecules possess a more planar conformation in the solid state than in solution. Thanks to fast (Foerster type) energy transfer processes, in 
the solid state the photoexcitations migrate towards the lower energy emitting states. These states are related to planar molecules possessing higher conjugation length and therefore an intrinsically higher QY. On the other hand, thanks to the steric hindrance of the phenyl groups, they do not feel the strong intermolecular interactions typical of unsubstituted oligomers. Therefore molecules $\mathbf{a}$ and $\mathbf{b}$ in the solid state behaves as quasi-isolated planar oligomers whose emission properties are not so different with respect to those of the solution phase.

In particular, molecule $\mathbf{b}$ shows good PL quantum yields, nearly independent on its aggregation state. This leads to the hypothesis that in this molecule the thiophene chain is short enough to allow the bulky groups to suppress the interaction of $\pi$-orbitals among adjacent molecules, but is sufficiently long to give a significative quantum yield. Its solid state QY of about $20 \%$ is comparable with the best reported in the literature for thiophene-based oligomers [4,15-17].

\section{CONCLUSIONS}

In this letter we report a study of the absorption and emission properties of a class of substituted oligothiophenes, highlighting the correlation between the optical response and the chemical structure of the materials. Steric hindered groups are linked to the thiophene chain in two different positions, $\beta$ or $\alpha$, giving respectively a twisted and a more planar conformation at the ground state.

From the optical features of the molecules in solution we conclude that when bulky groups are linked in the $\beta$ position of the thiophene chain the PL quantum yield decreases. For these molecules the large geometrical difference between the ground and the excited states, evidenced by the high Stokes shift, induces torsional relaxations during the photoexcitation process that open non-radiative decays channels.

When the bulky groups are linked in the $\alpha$ position, the molecules show optical features typical of oligothiophenes, that is red-shift of the emission and increase of the quantum yield as the thiophene chain grows. Moreover, the presence of the phenols gives raise to an enhancement of the quantum yield with respect to unsubstituted oligothiophenes and a reduction of PL quenching effects due to intermolecular interactions in the solid state. These findings have been related both to the increase of the $\pi$-conjugation, that is extended also on the phenyl rings, and to the steric effect of the substituents, that prevent H-type aggregation in the solid state. A significant photoluminescence quantum yield, compared with other thiophene-based oligomers, is obtained in particular for the bulky substituted molecule b. This molecule is a promising material for OLEDs because its PL quantum yield of about $20 \%$ is independent from the deposition method, thus assuring high repeatability during the film realization.

\section{EXPERIMENTAL}

Optical absorption and photoluminescence measurements were performed on dilute solutions $\left(<10^{-5} \mathrm{M}\right.$, absorbance below 0.2) in chloroform, $n$-heptane or THF (ACS reagent). Optical absorption measurements were performed using a Perkin-Elmer Lambda-9 spectrometer. Photoluminescence continuous wave measurements were obtained with a SPEX $270 \mathrm{M}$ monochromator equipped with a $\mathrm{N}_{2}$ cooled $\mathrm{CCD}$, and excitation with a monochromated Xe lamp; samples were excited at their absorption maxima except for the measurement of solution quantum yields. Photoluminescence quantum yields in solution were measured using quinine sulfate as a reference; the excitation wavelength was chosen among $313 \mathrm{~nm}$ and $365 \mathrm{~nm}$, depending on the absorption maxima of the sample. Photoluminescence quantum yields on solids were measured using a home-made integrating sphere following the procedure reported in [13], and a monochromated Xe lamp. The QY values were obtained as averages of measurements performed on analogous samples.

The spin coated films were obtained with a spin-coater LAURELL WS-400B-6NPP lite (1000 rpm).

DFT calculations were done on a cluster Xeon -Exadron at CILEA (Consorzio Interuniversitario Lombardo per l'Elaborazione Automatica). The software was Gaussian 03 [18]. The functional B3LYP and the basis set $6-31 \mathrm{G}^{* *}$ were used.

\section{ACKNOWLEDGEMENTS}

This work has been partly supported by the Italian MIUR through FIRB project RBNE033KMA and through FIRB project RBNE03S7XZ.

\section{REFERENCES}

[1] Ponomarenko SA, Kirchmeyer S, Elschner A, et al. Decyl-end capped thiophene-phenylene oligomers as organic semiconducting materials with improved oxidation stability. Chem Mater 2006; 18 (2): 579-586.

[2] Lee SA, Hotta S, Nakanishi F. Spectroscopic characteristics and intermolecular interactions of thiophene/phenylene co-oligomers in solutions. J Phys Chem A 2000; 104 (9):1827-1833.

[3] Cornil J, dos Santos DA, Crispin X, Silbey R, Bredas JL. Influence of Interchain Interactions on the Absorption and luminescence of conjugated oligomers and polymers: a quantum-chemical characterization. J Am Chem Soc 1998; 120:1289.

[4] Noda T, Ogawa H, Noma N, Shirota Y. A novel family of amorphous molecular materials containing an oligothiophene moiety as color-tunable emitting materials for organic electroluminescent devices. Adv Mater 1997; 9: 720.

[5] Noda T, Imae I, Noma N, Shirota Y. 5,5"-Bis(4-[bis(4methylphenyl)amino]phenyl\}-2,2':5',2"-terthiophene and 5,5"'$\operatorname{Bis}(4-[b i s(4-m e t h y l p h e n y l) a m i n o] p h e n y l\}-2,2^{\prime}: 5^{\prime}, 2: 5^{\prime}, 2^{\prime \prime}$ quaterthiophene as a novel family of amorphous molecular materials. Adv Mater 1997; 9: 239.

[6] Karpe S, Cravino A, Frere P, Allain M, Mabon G, Roncali J. 3D $\pi$ Conjugated oligothiophenes based on sterically twisted bithiophene nodes. Adv Funct Mater 2007; 17:1163-1171.

[7] Takahashi K, Suzuki T, Akiyama K, Ikegami Y, Fukazawa Y. Synthesis and characterization of novel para-terphenoquinone analogs involving a central dihydrothiophenediylidene structure. J Am Chem Soc 1991; 113: 4576.

[8] Takahashi K, Gunji A, Yanagi K, Miki M. Synthesis of novel heteroquaterphenoquinones and their electrochemical, structural, and spectroscopic characterization. J Org Chem 1996; 61(14): 47844792.

[9] D'Amore F, Lanata M, Gallazzi MC, Zerbi G. Optical properties of a quinoid molecule. Chem Phys Lett 2003; 377: 243-248.

[10] Lakowicz JR. Principles of fluorescence spectroscopy. Kluwer Academic Publishing/Plenum: New York; 1999.

[11] Benincori T, Bongiovanni G, Botta C, et al. Tuning of the excited state lifetime by control of the structural relaxation in oligothiophenes. Phys Rev B 1998; 58: 9082.

[12] Becker RS, De Melo JS, Macuanita AL, Elisei F. Comprehensive evaluation of the absorption, photophysical, energy transfer, structural, and theoretical properties of $\alpha$-oligothiophenes with one to seven rings. J Phys Chem 1996; 100: 18683-18695. 
[13] De Mello JC, Wittmann HF, Friend RH. An improved experimental determination of external photoluminescence quantum efficiency. Adv Mater 1997; 3: 230-232.

[14] Botta C, Destri S, Pasini M, et al. Chromophores in nanochannels for energy transfer and conversion. Synth Met 2003; 139: 791.

[15] Mazzeo M, Vitale V, Della Sala F, et al. New branched thiophenebased oligomers for bright organic light-emitting devices. Adv Mater 2003; 24(15): 2060-2063.
[16] Lee TH, Tong KL, So SK, Leung LM. Synthesis and electroluminescence of thiophene-based bipolar small molecules with different arylamine moieties. Synt Met 2005; 155: 116-124.

[17] Borshchev OV, Ponomarenko SA, Surin NM, et al. Bithiophenesilane dendrimers: synthesis and thermal and optical properties. Organometallics 2007; 26: 5165-5173.

[18] Frisch MJ, Trucks GW, Schlegel HB, et al. Gaussian 03, Revision C.02. Gaussian Inc: Wallingford CT; 2004.

Received: December 23, 2007

(C) Canesi et al.; Licensee Bentham Open.

This is an open access article distributed under the terms of the Creative Commons Attribution License (http://creativecommons.org/license/by/2.5/), which permits unrestrictive use, distribution, and reproduction in any medium, provided the original work is properly cited. 